\title{
Cefcapene Pivoxil Hydrochloride Hydrate
}

National Cancer Institute

\section{Source}

National Cancer Institute. Cefcapene Pivoxil Hydrochloride Hydrate. NCI Thesaurus.

Code C98219.

The hydrate hydrochloride salt form of cefcapene pivalate, a prodrug and thirdgeneration cephalosporin with antibacterial activity. After oral administration of cefcapene pivoxil hydrochloride hydrate the ester bond is cleaved, releasing active cefcapene. 